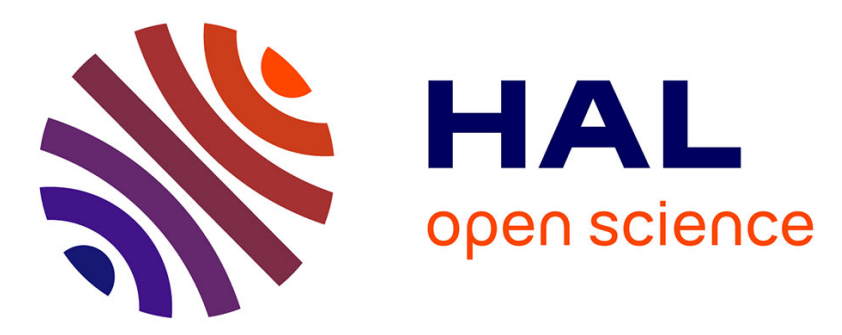

\title{
Laser-induced-fluorescence observation of ion velocity distribution functions in a plasma sheath
}

N. Claire, G. Bachet, U. Stroth, F. Doveil

\section{To cite this version:}

N. Claire, G. Bachet, U. Stroth, F. Doveil. Laser-induced-fluorescence observation of ion velocity distribution functions in a plasma sheath. Physics of Plasmas, 2006, 13 (6), pp.062103. 10.1063/1.2206786 . hal-01787094

\section{HAL Id: hal-01787094 \\ https://hal.science/hal-01787094}

Submitted on 18 Apr 2019

HAL is a multi-disciplinary open access archive for the deposit and dissemination of scientific research documents, whether they are published or not. The documents may come from teaching and research institutions in France or abroad, or from public or private research centers.
L'archive ouverte pluridisciplinaire HAL, est destinée au dépôt et à la diffusion de documents scientifiques de niveau recherche, publiés ou non, émanant des établissements d'enseignement et de recherche français ou étrangers, des laboratoires publics ou privés. 


\title{
Laser-induced-fluorescence observation of ion velocity distribution functions in a plasma sheath
}

\author{
N. Claire and G. Bachet \\ Equipe Turbulence Plasma, LPIIM, CNRS-Université de Provence, Marseille, France \\ U. Stroth \\ Universität Stuttgart Institut für Plasmaforschung, Pfaffenwaldring, 31 D-70569 Stuttgart, Germany \\ F. Doveil \\ Equipe Turbulence Plasma, LPIIM, CNRS-Université de Provence, Marseille, France
}

(Received 6 September 2005; accepted 1 May 2006; published online 12 June 2006)

\begin{abstract}
Experimental results obtained by laser-induced-fluorescence on metastable ion velocity distribution functions (MIVDF) in electrostatic presheaths and sheaths in argon plasmas produced by the thermoionic effect in a multipolar dc discharge are presented. The shape of the measured MIVDF are in qualitative agreement, for the presheath, with Emmert's model and exhibit: (1) a Maxwellian profile at the center of the device where the potential is zero; (2) a distribution function's shape made of three distinct parts at the entrance of the presheath. Inside the sheath the recorded MIVDF recovers a Maxwellian profile with a width unexpectedly related to the background neutral pressure. The velocity and potential profiles that can be deduced from the measured MIVDF show a strong influence of the primary electrons emitted by the filaments. (C) 2006 American Institute of Physics.
\end{abstract}

[DOI: 10.1063/1.2206786]

\section{INTRODUCTION}

When a floating plate is introduced into a plasma, it gets negatively charged since the electron population is the most mobile species. A steady state is reached when the plate's potential is able to repel the electrons and such that the ion and electron currents on the plate are equal. In front of the plate a sheath is formed.

Since the work of Tonks and Langmuir ${ }^{1}$ the study of presheath and sheath potential has motivated many analytical (see, for example, Refs. 2-6) and numerical works (as given by Refs. 7-16). A review article on the Bohm criterion and sheath formation can be found in Ref. 17.

One way to experimentally obtain the ion velocity distribution functions in the presheath and sheath without disturbing it, is to use a diagnostic having a good spatial and velocity resolution. The laser induced fluorescence (LIF) diagnostic fulfills these requirements. Bachet et al. ${ }^{18}$ have earlier obtained presheath and sheath ion velocity distribution functions in a multipolar argon discharge essentially with the aim to show the diagnostic possibilities. In this paper, the spatial resolution was not optimized and the results were distorted by the convolution with the apparatus function as is shown in the paper itself and later on by Sheridan ${ }^{15}$ in his numerical simulations. The differences observed with the distribution functions, calculated from Emmert's model, ${ }^{5}$ may also be explained by this apparatus function. Other authors, ${ }^{19}$ have also measured the distribution functions in a presheath with LIF but their velocity resolution was not sufficient. Severn et al. ${ }^{20}$ have also measured the distribution functions in a mixture of two gases to observe how the Bohm criterion evolves. More recently, LIF has also been used to measure ion acceleration to supersonic speeds in double layers. $^{21}$
The aim of this paper is to repeat the measurement of the distributions in the presheath and in the sheath of a floating plate inserted in a multipolar argon plasma discharge with an apparatus function small enough to have negligible effects on the results, in order to compare them to Emmert's analytical model. This model is the most useful for comparisons because, to our knowledge, it is the only one that determines the distribution functions in the presheath beside predicting the velocity and potential profiles.

The paper is organized as follows: Sec. II is devoted to a brief recall of Emmert's model. ${ }^{5}$ Section III describes the experimental setup. Section IV presents the results and Sec. $\mathrm{V}$ gives our conclusion.

\section{EMMERT’S MODEL}

A complete description of the model can be found in the original paper. $^{5}$

This model describes a plasma created in volume, like the one resulting from a multipolar thermoelectronic discharge. It is a 1D model based on a kinetic description of the ions. The main differences between this model and our experiment is that, in a multipolar device, two electron populations are present:

- One resulting from the filament electronic emission accelerated by the potential discharge and creating the plasma by collision with the neutral. This population of "primary electrons," 12 probably has a spatial isotropic drift velocity around $11 \mathrm{eV}$ and a velocity spread of about $5 \mathrm{eV}$;

- The other, formed by the bulk electrons coming from neutral gas ionization and having a temperature around $2 \mathrm{eV}$ in our plasma conditions. 


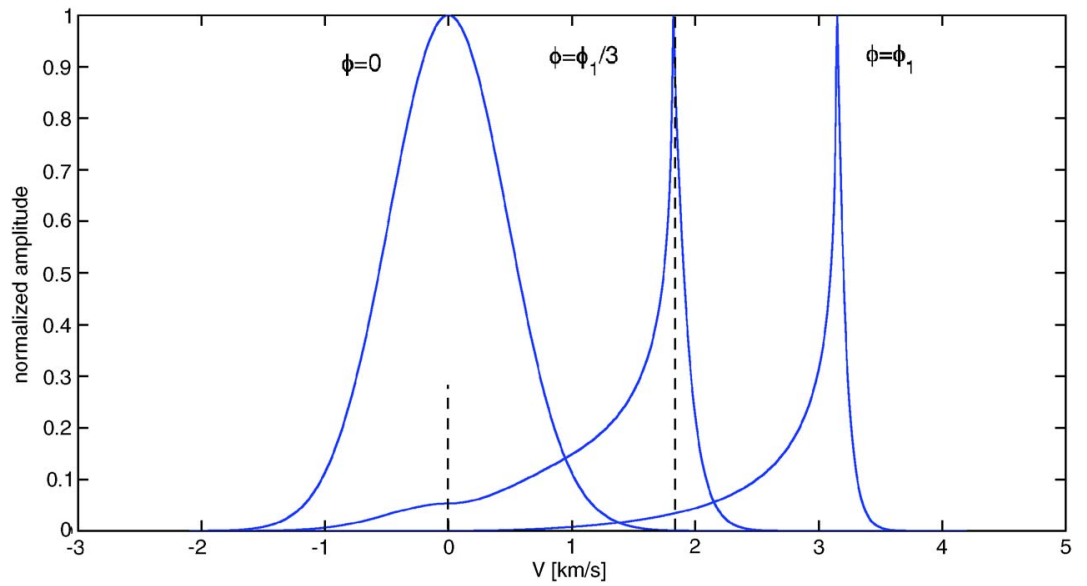

FIG. 1. Emmert's model for ion velocity distribution functions for $\phi=0, \frac{\phi_{1}}{3}, \phi_{1}$. Dashed lines limit the three parts of the distribution function for the case $\phi=\frac{\phi_{1}}{3}$.
Simulations with two negative Boltzmann components, as given by Refs. 13 and 15, conclude, in these peculiar plasma conditions, to the presence of a double layer in the sheath.

In Emmert's model only one electronic temperature is taken into account and the electronic density follows the Boltzmann relation:

$$
n_{e}(x)=n_{e 0} \exp \left[\frac{e \phi(x)}{k T_{e}}\right],
$$

where $n_{e 0}$ is the background plasma density, $T_{e}$ is the electron temperature, and $\phi(x)$ is the local potential assumed to be zero in the bulk plasma.

The energy source function yields, as pointed out by Procassini, ${ }^{8}$ a velocity distribution source function of the "flux" type:

$$
S_{i}\left(x, v_{x}\right)=\frac{h(x) m_{i} v_{x}}{k T_{i}} \exp \left[\frac{-m_{i} v_{x}^{2}}{2 k T_{i}}\right],
$$

where $h(x)$ is the "spatial ion source" intensity profile, $T_{i}$ is the ion temperature, $m_{i}$ is the ion mass. This equation means that there is no ion created with zero velocity.

With these assumptions, the electrostatic potential and the ion velocity distribution function can be obtained in the presheath. To each position in the presheath is associated with a potential $\phi$ up to a certain potential value $\phi_{1}{ }^{5}$ The plate potential $\phi_{w}$ can also be calculated with this model. Obviously this wall potential is not the one measured in our device since it is fixed by the most energetic species reaching the plate, i.e., the primary electrons, with energy resulting from both the discharge potential and the neutral pressure.

Figure 1 shows three distribution functions for different potentials: $\phi=0 ; \frac{\phi_{1}}{3} ; \phi_{1}$ monotonously associated with three different positions.

A distribution function at any given point $x_{0}$ consists of three parts:

- a left part which is half a Gaussian distribution centered on zero velocity due to the ions created in region I of Fig. 2, with a negative velocity;

- a right part which is also a part of a Gaussian distribution centered on zero velocity but truncated at $v_{0}$, where $v_{0}$ is the velocity of an ion initially at rest on top of the potential hill and accelerated to $x_{0}$. This part of the distribution results from the acceleration by the electrostatic field of ions created in region IIa with a positive velocity and of ions created in region IIb having enough energy to climb the potential hill;

- a middle part region which connects the two others and which is made of ions created in I and IIa with negative velocity but reflected by the potential in region IIa and ions created in IIa with a velocity less than $v_{0}$ even after being accelerated by the potential gradient.

\section{EXPERIMENTAL SETUP}

Experiments are performed in a multipolar ArII plasma device. The $40 \mathrm{~cm}$ diam, $80 \mathrm{~cm}$ long cylindrical plasma is ended by two conducting plates at floating potential. At about $8 \mathrm{~cm}$ from the device cylindrical wall, the plasma can be considered as unmagnetized ${ }^{22}$ and no permanent magnet is installed on both ends. One plate has a hole of diameter $10 \mathrm{~mm}$ to allow the laser beam to enter into the plasma, the other one holds a $5 \mathrm{~cm}$ squared plate covered by a layer absorbing the laser beam to avoid its reflection. The plasma is produced by thermoelectronic discharge; the plasma parameters are shown in Table I and a sketch of the device is given in Fig. 3.

The LIF diagnostic uses a cw ring dye vertically linearized laser from coherent chopped by an external acoustooptic modulator (AOM). The emitted laser line, whose frequency can be manually fixed anywhere in the dye tuning range, has a bandwidth of $0.5 \mathrm{MHz}$ much less than the natu-

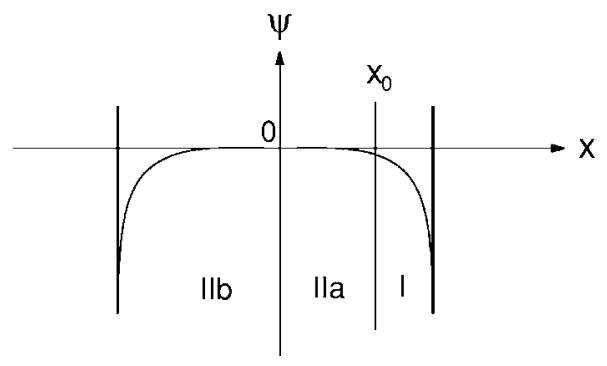

FIG. 2. Emmert's assumed potential and the three regions (I, IIa, and IIb) involved in the description of the distribution function in Emmert's model. 
TABLE I. Parameters of the experiment. $T_{e}$ and $n_{e}$ are measured by the Langmuir probe, $T_{i}$ is measured by LIF.

\begin{tabular}{lc}
\hline \hline Parameter & Value \\
\hline$T_{e}$ & $2.5 \mathrm{eV}$ \\
$T_{i}$ & $0.027 \mathrm{eV}$ \\
Secondary $n_{e}=n_{i}$ & $5.510^{9} \mathrm{~cm}^{-3}$ \\
Primary $n_{p}$ & $210^{6} \mathrm{~cm}^{-3}$ \\
Pressure & $1.810^{-4} \mathrm{mbar}$ \\
Discharge current & $0.5 \mathrm{~A}$ \\
Discharge potential & $50 \mathrm{~V}$ \\
\hline
\end{tabular}

ral linewidth of the transition used $(14 \mathrm{MHz}$, this natural linewidth determines the maximum velocity resolution of the diagnostic). The laser line can scan a frequency interval which can reach $30 \mathrm{GHz}$. The laser beam can be propagated through the plasma, either perpendicularly (axial direction of the device) or parallel (transversal direction) to the plate. The fluorescence light induced by the laser beam is collected along the radial direction of the cylindric plasma by an optical objective and an image of magnitude of the laser beam is formed on a slit-diaphragm which fixes the spatial resolution of the diagnostic. A $0.125 \mathrm{~mm}$ slit is used to reduce the effect of the apparatus function.

A $1 \mathrm{~nm}$ bandpass optical filter isolates the fluorescence light which is detected by a photomultiplier tube (PMT). The PMT pulses are amplified and counted by a multichannel scalar (MCS) which sets the time resolution.

The three argon atomic levels used for the laser induced fluorescence are the $3 d^{2} G_{9 / 2}$ metastable one which is laser excited to the $4 p^{2} F_{7 / 2}$ level $(611 \mathrm{~nm})$ with observed decay to $3 s^{2} G_{9 / 2}$ (460.9 nm) (for a reference on collisional deexcitation and metastable lifetimes, see Ref. 23).

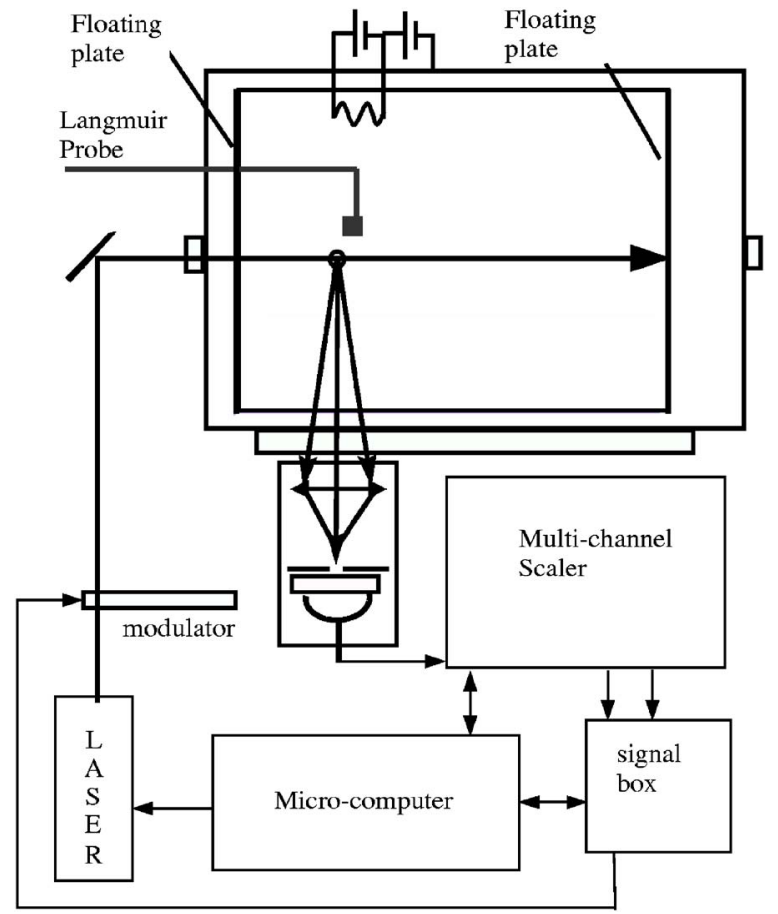

FIG. 3. Experimental setup: the multipolar device, the acquisition system, optics, and the laser beam in the longitudinal position.

\section{RESULTS}

To optimize our measurements, we first study the laser power broadening.

Figure 4 shows, for three different laser powers, the ion transverse velocity distribution functions (ITVDF, velocity component parallel to the plate) recorded near the center of the device (maximum velocity near $0 \mathrm{~km} / \mathrm{s}$ ). We observe that increasing the laser power from $25 \mathrm{~mW}$ to $250 \mathrm{~mW}$ produces a spurious broadening of the ITVDF. To avoid this

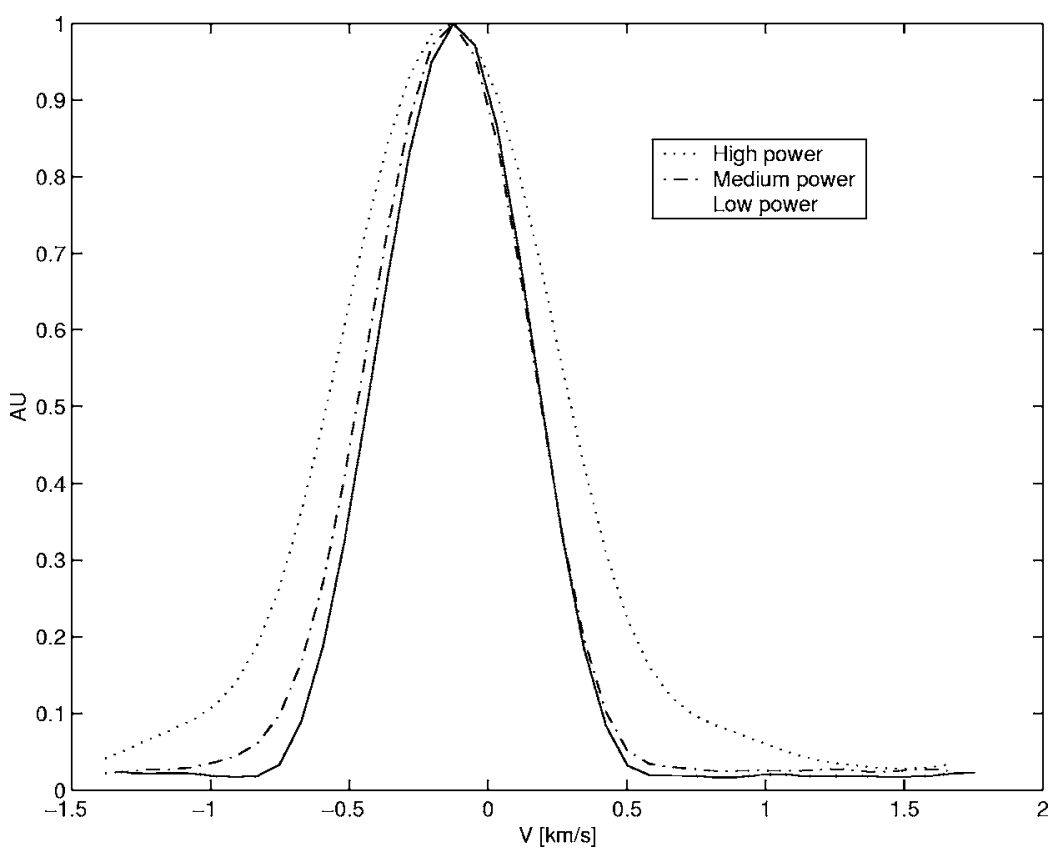

FIG. 4. Laser power effect on the ion longitudinal velocity distribution function. 


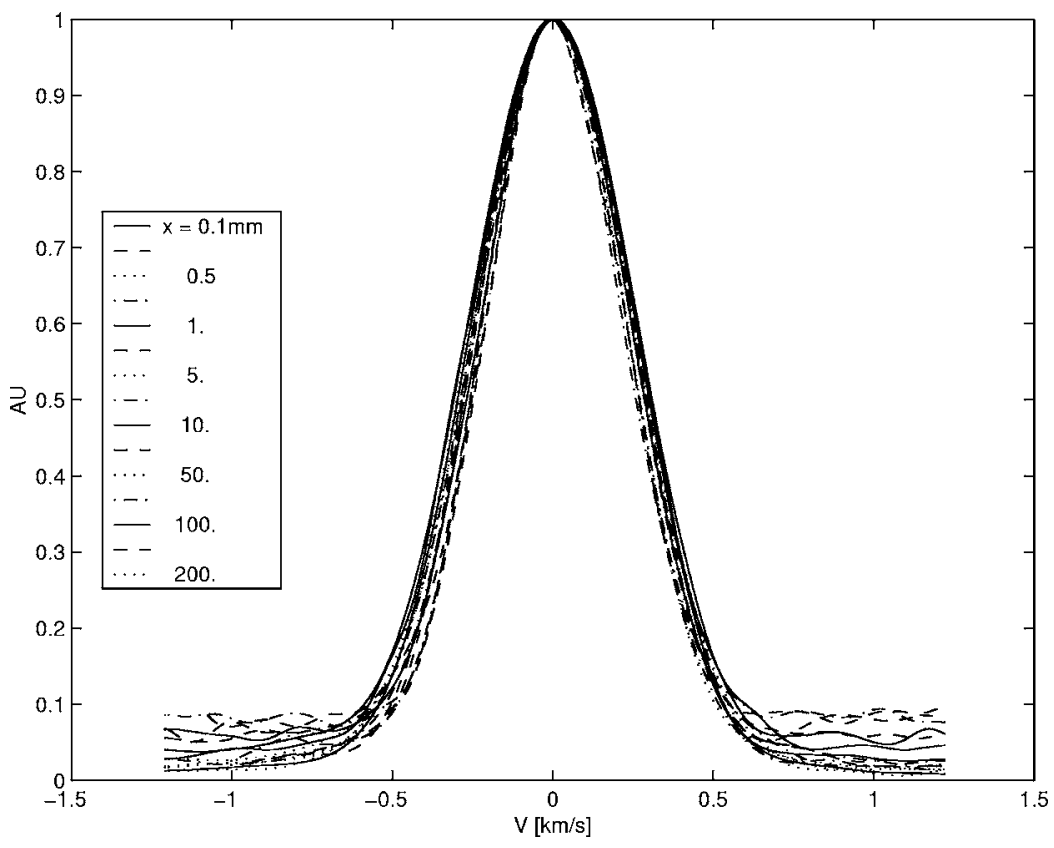

FIG. 5. Ion transversal velocity distribution functions at several positions for a neutral pressure equal to 4.8 $\times 10^{-4}$ mbar. broadening effect the laser power is reduced to $25 \mathrm{~mW}$ by rotating a linear polarizer.

Then to assert, for comparison with Emmert's model, the one dimensionality of our plasma, we record amplitude normalized ITVDF for several locations in front of the plate as shown in Fig. 5. By definition $x=0 \mathrm{~mm}$ is the location of the plate located $20 \mathrm{~cm}$ from the end wall in the unmagnetized plasma. These ITVDF can be fitted by a Maxwellian distribution having an ion temperature around $0.027 \mathrm{eV}$ (the room temperature) for all spatial locations, even in the sheath. Because the laser beam is not exactly parallel to the plate for all the scanned positions, a negligible width variation, $\approx 50 \mathrm{~m} / \mathrm{s}$, can be observed. An ion longitudinal temperature equal to the transversal one can only be measured in the center of the device where the distribution function is not perturbed by the sheath potential.

Now the influence of the slit diaphragm on distributions is studied. The sheath is a region where the potential gradient is large. Therefore the longitudinal distributions exhibit a strong velocity shift. The velocity at the maximum of the ion longitudinal velocity distribution functions (ILVDF, velocity component perpendicular to the plate) $V$ is related, to first approximation, to the local potential by the energy conservation law $\frac{m_{i} V^{2}}{2}=-e \phi$. If we consider the evolution of $V$ versus $x$, and if $\Delta x$ is the slit width, we can associate a velocity error $\Delta V$ to the spatial uncertainty $\Delta x$. The bigger the potential gradient, the bigger $\Delta V$. In the sheath, the error will be big-

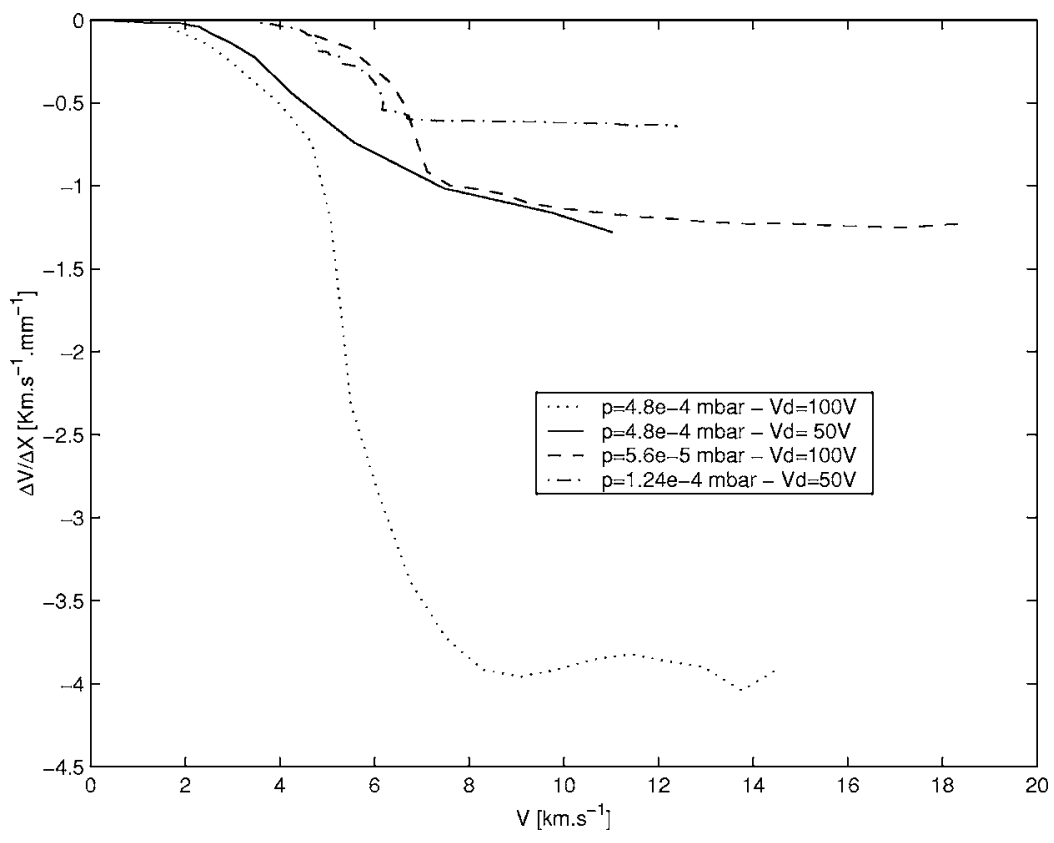

FIG. 6. Slope of the ion velocity profiles from the center of the plasma to the sheath for three pressure and two discharge potential values. 


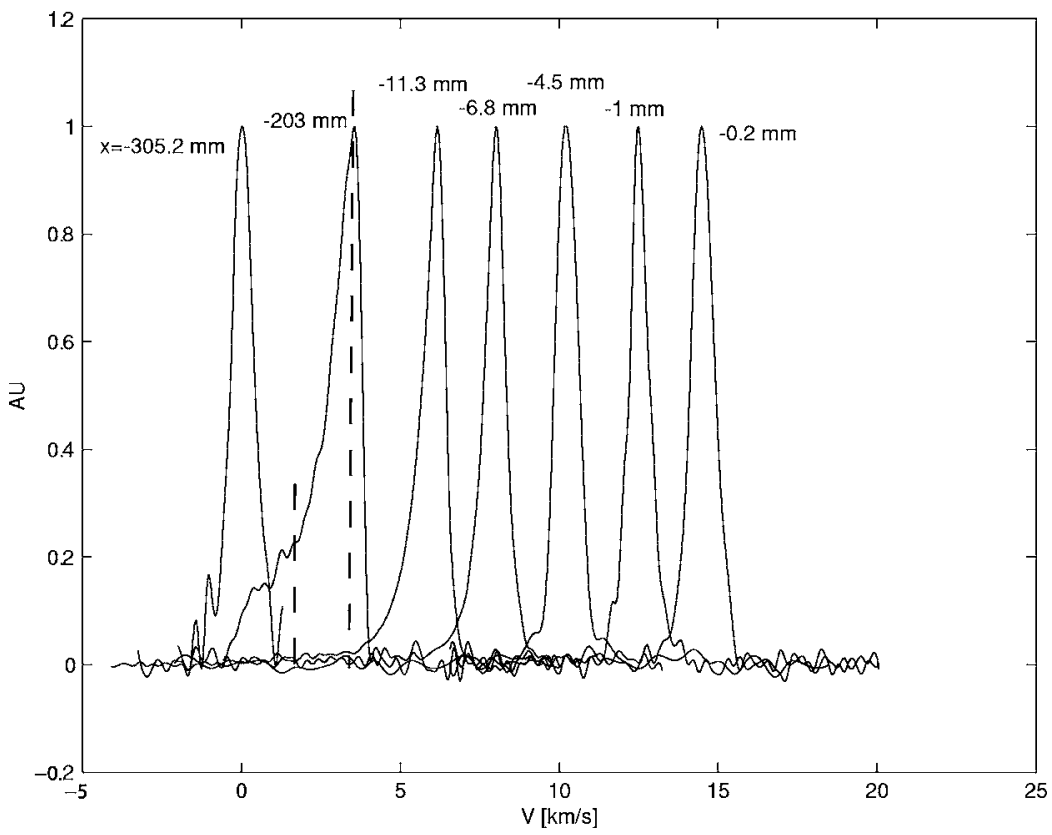

FIG. 7. Ion longitudinal velocity distribution functions of the plasma from the center of the device to the sheath obtained with a discharge potential equal to $50 \mathrm{~V}$ and a neutral pressure equal to $1.24 \times 10^{-4}$ mbar. Dashed lines show, for example, the three parts of one of the distribution functions. ger than in the presheath (in the center of the device, the gradient is zero, therefore $\Delta V$ is zero). In Fig. 6, we plot $\frac{\Delta V}{\Delta x}$ versus $V$ to estimate the velocity error due to the slit diaphragm. One has to keep in mind that a positive velocity ion goes away from the plate. In Fig. 6, it appears clearly that, for the slit's width $\Delta x=0.125 \mathrm{~mm}$, the velocity error is negligible, $\Delta V \approx 100 \mathrm{~m} / \mathrm{s} \ll v_{\text {th }}$ or $V$, and the velocity distributions are no more convoluted by an apparatus function, even in the sheath, except for the special plasma condition $P$ $=4.8 \times 10^{-4}$ mbar and discharge potential $V_{d}=100 \mathrm{~V}$. This was not the case in the earlier paper by Bachet et al. ${ }^{18}$

We are now ready to study the way ILVDF evolves in the sheath.

Figures 7 and 8 show typical ILVDF with their ampli- tude normalized to one for two different experimental conditions.

As it can be observed, the shape of the recorded ILVDF evolves from a Maxwellian in the center of the device to a distribution made of three parts as for Emmert's model, with, however, a difference.

Contrary to what is seen in this model where the left part of the distribution function is a half-Maxwellian truncated at its maximum occurring at $v=0 \mathrm{~km} / \mathrm{s}$, the left part looks like a drifting half-Maxwellian truncated at its maximum but at a drift velocity depending on plasmas parameters and on the spatial position where the measurements are taken. A study must be done on this difference between measurements and Emmert's model. This drift velocity is also observed on the

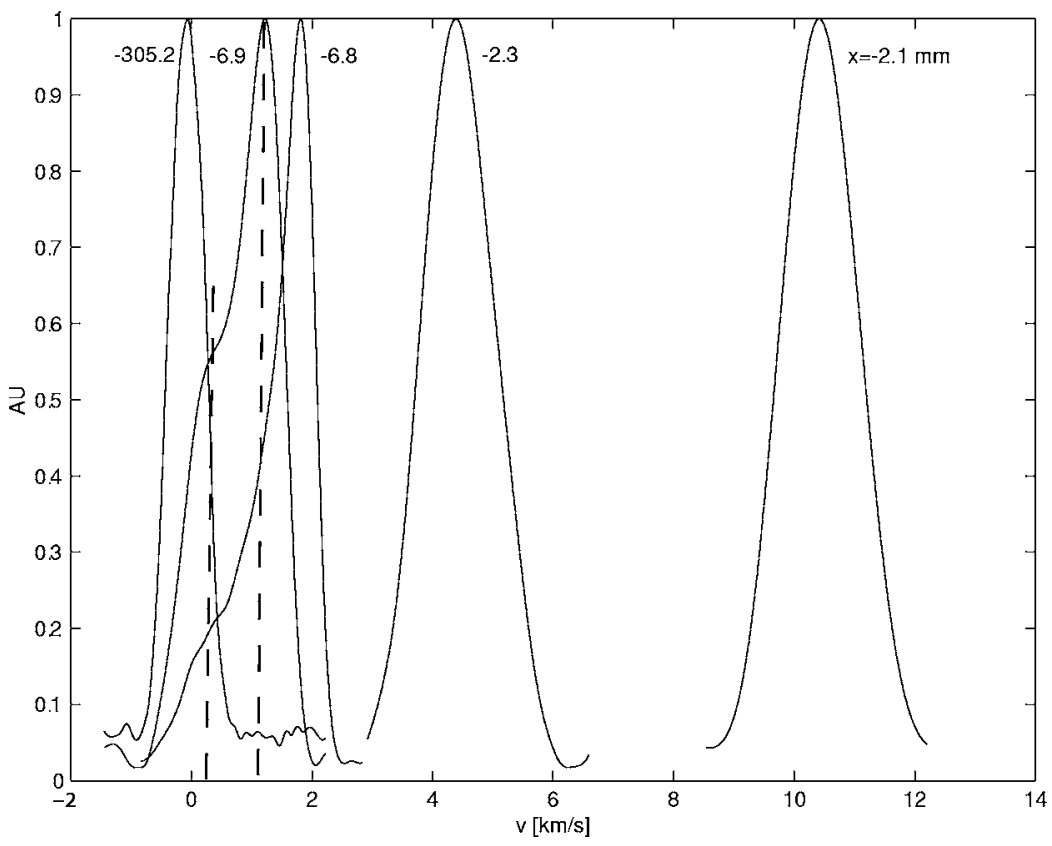

FIG. 8. Ion longitudinal velocity distribution functions of the plasma from the center of the device to the sheath obtained with a discharge potential equal to $100 \mathrm{~V}$ and a neutral pressure equal to $4.8 \times 10^{-4}$ mbar. Dashed lines show, for example, the three parts of one of the distribution functions. 


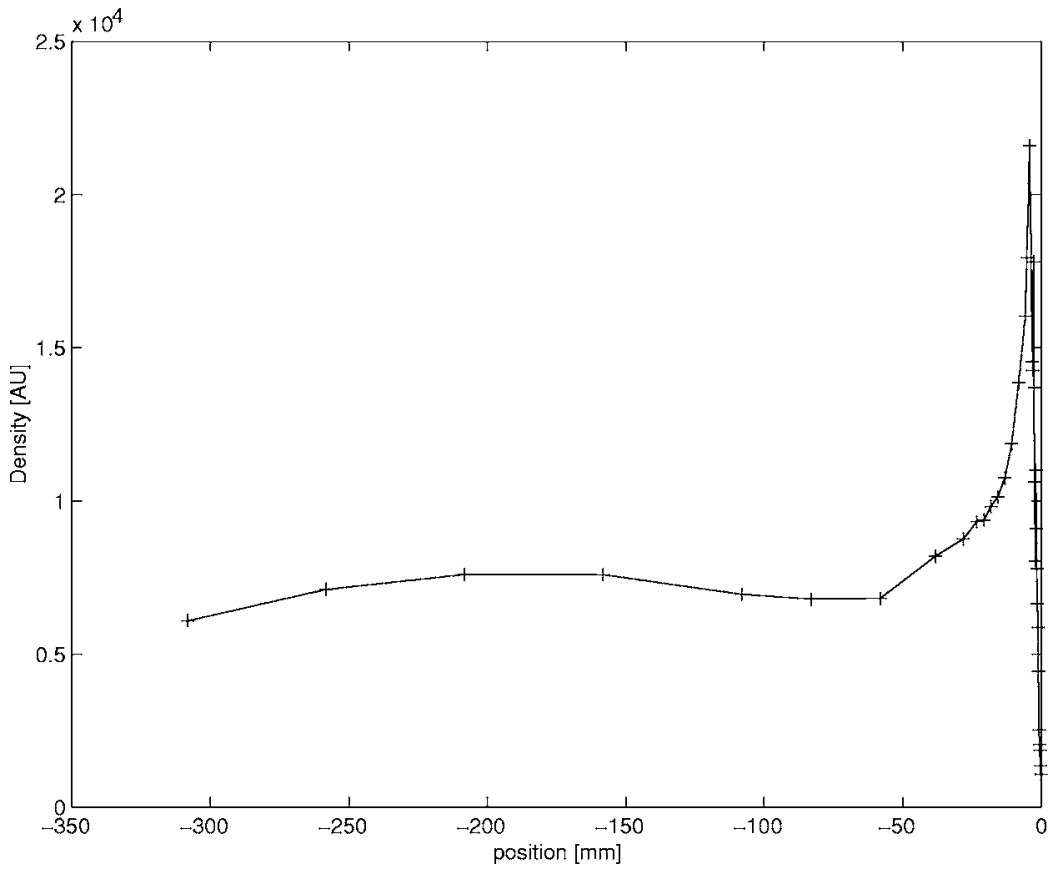

FIG. 9. Ion metastable density profiles from the center of the plasma to the sheath for a neutral pressure equal to $4.8 \times 10^{-4} \mathrm{mbar}$ and a discharge potential equal to $100 \mathrm{~V}$.
ILVDF obtained when the coupled Vlasov-Poisson equations are solved numerically. ${ }^{15,16}$

Closer to the wall we recover an asymmetric distribution function similar to Emmert's model.

In the sheath, the ILVDF evolves to a shape which can again be fitted by a Maxwellian curve, but can no longer be compared to Emmert's model which is not valid in the sheath. This Maxwellian profile can be interpreted as the result of a "thermalization" of the ILVDF in the sheath. This surprising result has never been observed, neither simulated before.

By integrating the ILVDF corresponding to a discharge potential of $50 \mathrm{~V}$ and a neutral pressure of $4.8 \times 10^{-4} \mathrm{mbar}$ measured with an ionization pressure gauge, we get the rela- tive ion metastable density profile showing an increase of the metastable density close to the plate, as can be seen in Fig. 9. From Fig. 10 we see that the position of the peak in the metastable density corresponds to a velocity equal to $2 \mathrm{~km} / \mathrm{s}$ close to ion acoustic speed, a criterion usually used to define the sheath entrance. Metastable ions are created by inelastic collisions of ions with low energy electrons (one has to keep in mind that LIF diagnostic only observes metastable ions). One possible explanation for the formation of the observed bump in metastable could be the enhancement of metastable creation (probably related to the form of the unknown cross section function as a function of electron energy) by reflected secondary electrons at the observed position. Stark effect can be neglected since electric field in the sheath is too small.

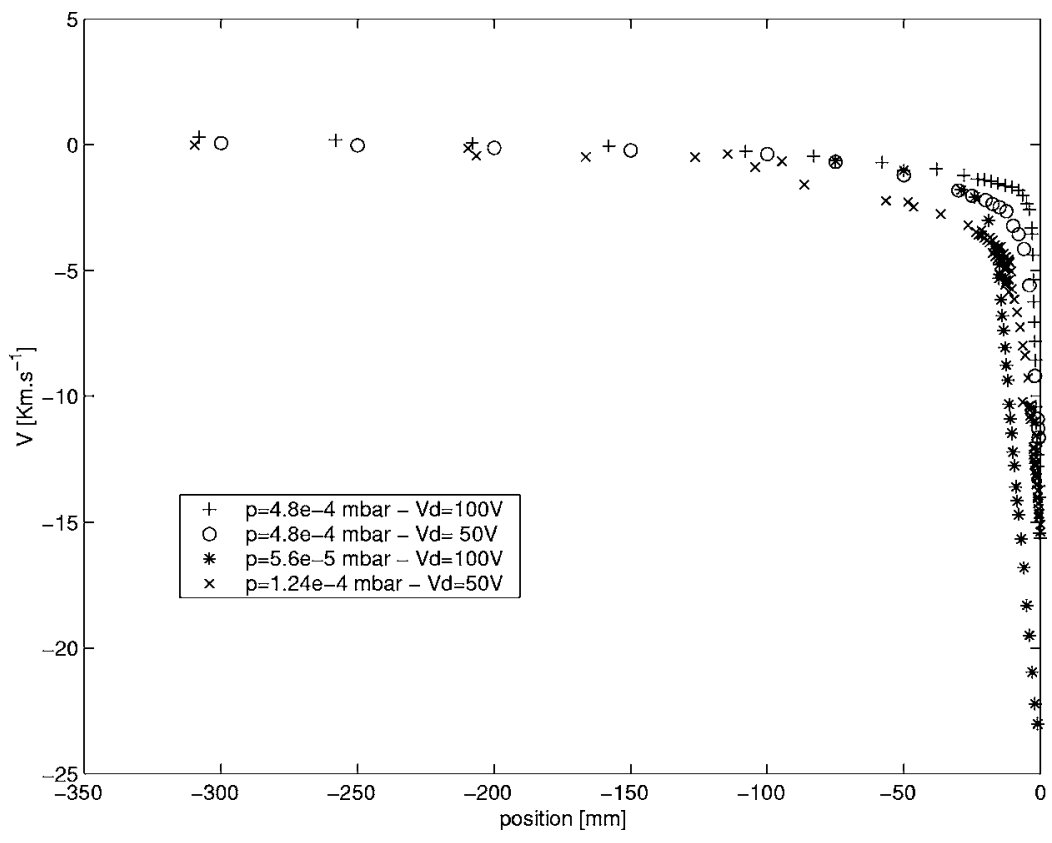

FIG. 10. Ion velocity profiles from the center of the plasma to the sheath for three neutral pressure values and two discharge potential values. 


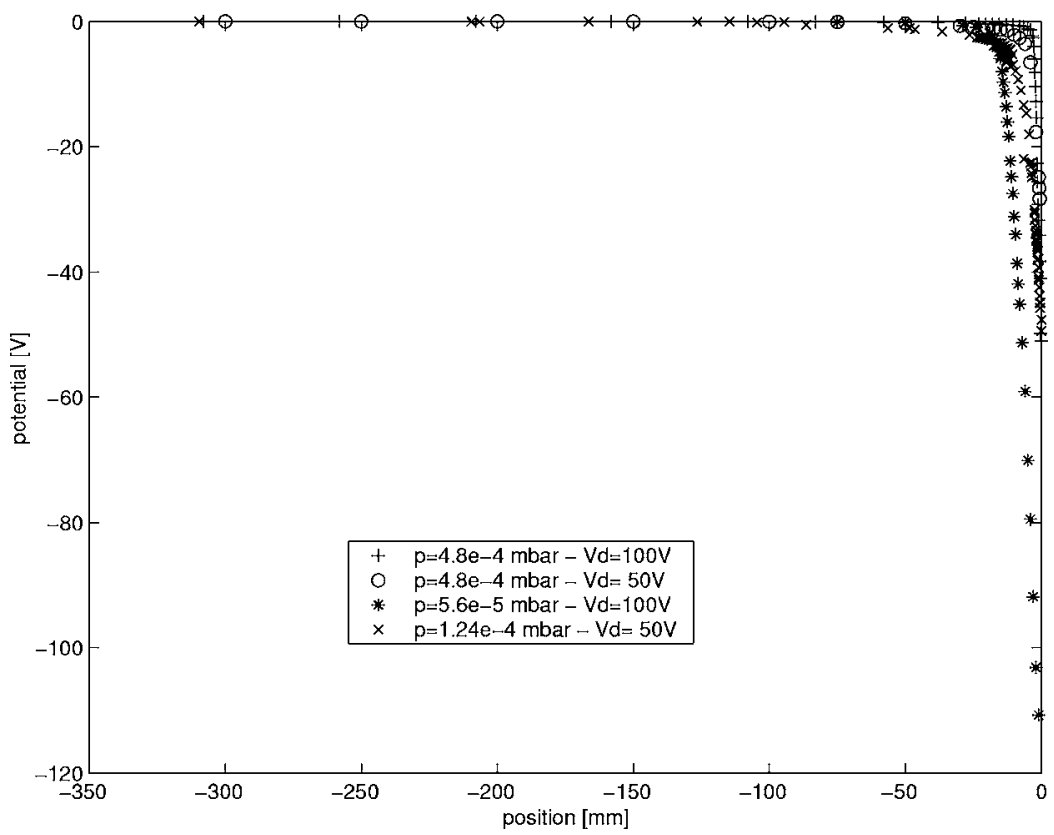

FIG. 11. Plasma potential profiles from the center of the plasma to the sheath for different plasma conditions.

Using the velocity at the maximum of the ILVDF we have obtained the velocity profile, Fig. 10, and the potential profile, Fig. 11, for three neutral pressures and two discharge potentials. The potential is calculated from energy conservation law and the reference potential is equal to the plasma potential in the center of the device.

We tried to obtain nondimensional curves from the four sheath potentials corresponding to different plasma conditions, by dividing each potential profile by its minimum magnitude and normalizing the positions by the estimated Debye length $\lambda_{D}$. The results are shown in Fig. 12. We see that the resulting two sets of curves we obtain are essentially depending on the discharge potential, therefore on the primary electron energy. The pressure plays only a secondary role. This analysis shows the importance of primary electrons and/or of the way to produce plasma on the sheath behavior even though their density is very low as shown in Table I.

In Fig. 13 are reported the distribution functions recorded with a discharge potential of $50 \mathrm{~V}$, for several neutral argon pressures having their maximum at quite the same velocity $\left(v_{\text {drift }}\right.$ around $\left.3.5 \mathrm{~km} / \mathrm{s}\right)$ but not at the same spatial location. We may assume that the potential at these recorded points is almost the same for all the measurements. In this figure, the curves have been shifted so that their maximum is located at the same place (denominated by $v=0$ ). Following Emmert's model, these distributions should be the same since their velocity drift (or potential where they are re-

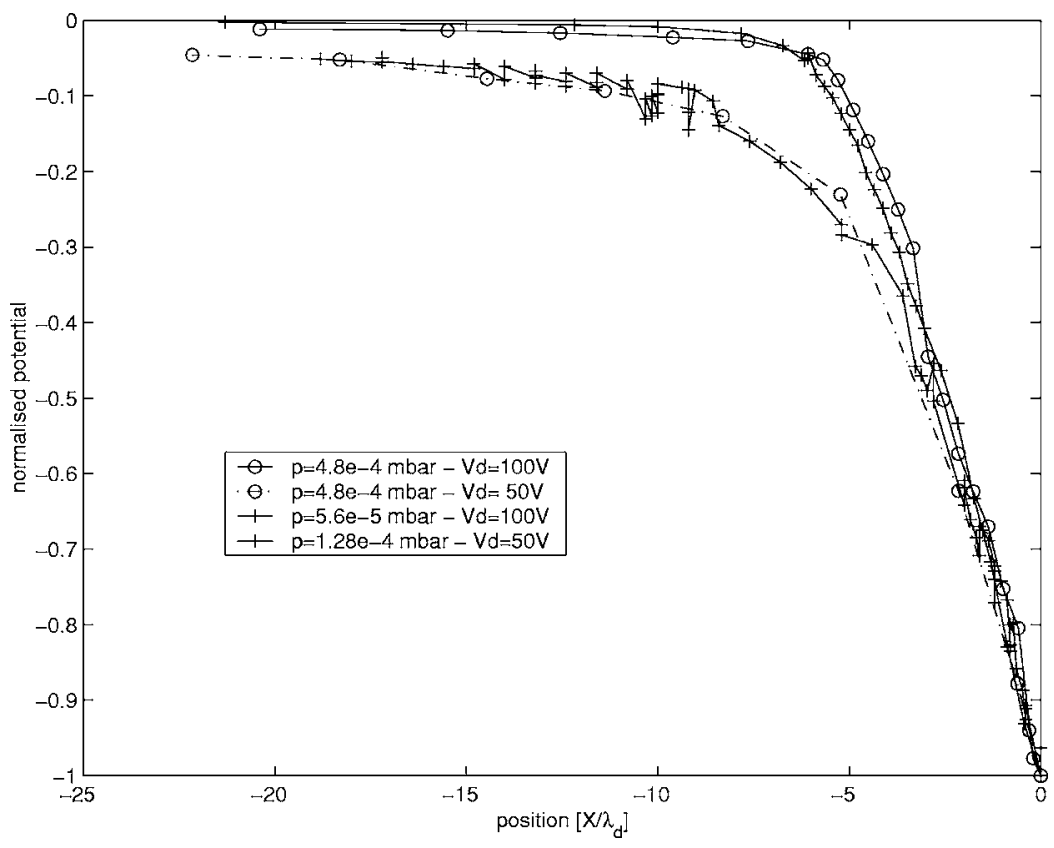

FIG. 12. Normalized plasma potential profiles from the center of the plasma to the sheath. 


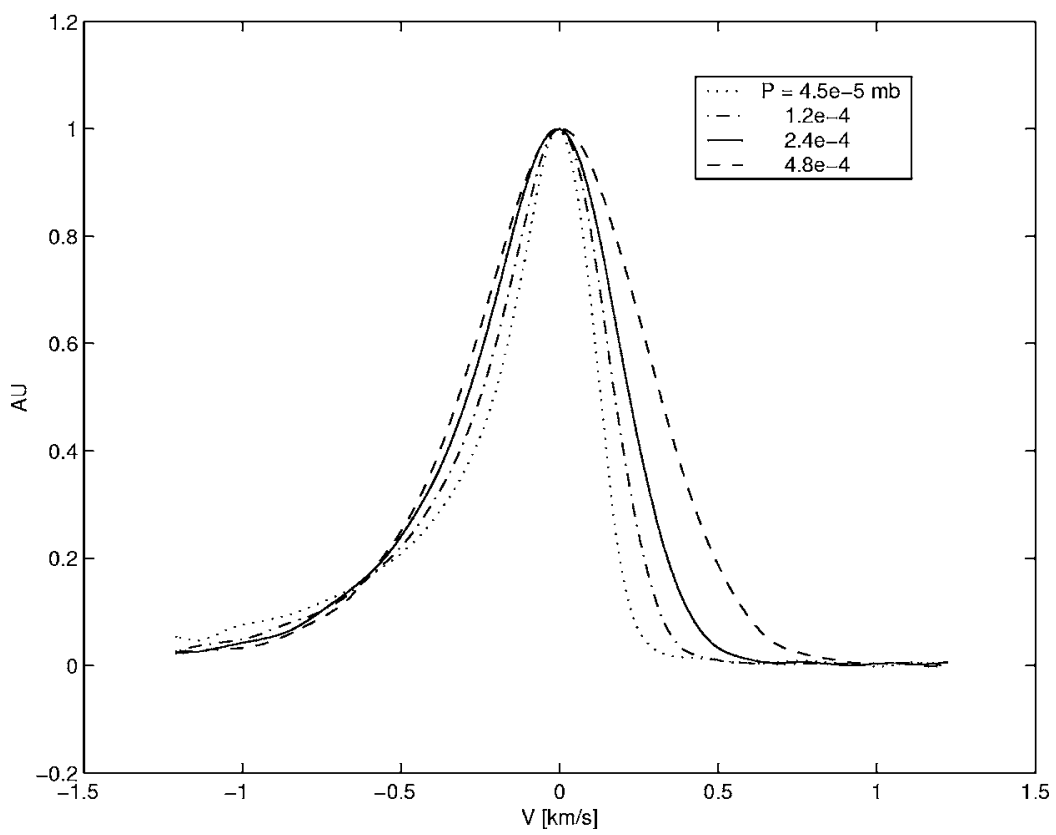

FIG. 13. Pressure effect on the ion longitudinal velocity distribution function at the entrance of the sheath. The ions mean velocity is around $3.5 \mathrm{~km} / \mathrm{s}$. corded) is identical. In fact it can be observed that, in the sheath, when the pressure increases, the width of the distribution functions increases.

\section{CONCLUSION}

New experimental results on electrostatic sheaths have been obtained in a widely used unmagnetized multipolar argon plasma. The velocity and potential profiles have been determined in condition of no convolution by an apparatus function. The power broadening of the ILVDF, even when a cw dye laser is used, has been observed and controlled.

The ILVDF recorded in the presheath are in qualitative agreement with the simple 1D Emmert's model and exhibit:

- a Maxwellian profile at the center of the device where the potential is zero, with an ion temperature equal to the ambient temperature $(0.027 \mathrm{eV})$. This is also the temperature measured from the ITVDF;

- a shape composed of three pieces in the presheath, but with the first part shifted as compared to Emmert's model, probably due to the presence of primary electrons in our experiment.

It is reasonable to expect that the result displayed by Fig. 12 can be interpolated to any discharge potential value in the explored range and we can therefore conclude that primary electrons play a significant role in the potential behavior and sheath formation. Ion collisions with repelled electrons is our hypothesis to explain the spatial increase of ion metastable density observed near the sheath entrance.

Despite the small thickness of the sheath as compared to the neutral-ion mean free path, an unexpected neutral pres- sure effect on the distributions' width is observed inside the sheath.

These new effects on an old plasma physics problem should trigger new theoretical, numerical, and experimental efforts to elucidate their origin.

${ }^{1}$ L. Tonks and I. Langmuir, Phys. Rev. 34, 876 (1929).

${ }^{2}$ E. R. Harrison and W. B. Thompson, Proc. Phys. Soc. London 74, 145 (1959).

${ }^{3}$ L. S. Hall, Proc. Phys. Soc. London 80, 309 (1962).

${ }^{4}$ A. Caruso and A. Cavaliere, Nuovo Cimento 26, 1389 (1962).

${ }^{5}$ G. A. Emmert, R. M. Wieland, A. T. Mense, and J. Davidson, Phys. Fluids 23, 903 (1980).

${ }^{6}$ R. C. Bissel and P. C. Johnson, Phys. Fluids 30, 2264 (1987).

${ }^{7}$ S. A. Self, Phys. Fluids 6, 1762 (1963).

${ }^{8}$ R. J. Procassini, C. K. Birdsall, and E. C. Morse, Phys. Fluids B 2, 3191 (1990).

${ }^{9}$ L. A. Schwager and C. K. Birdsall, Phys. Fluids B 2, 1057 (1990).

${ }^{10}$ T. E. Sheridan and J. Goree, Phys. Fluids B 3, 2796 (1991).

${ }^{11}$ J. M. Grossman, S. B. Swanekamp, and P. F. Ottinger, Phys. Fluids B 4, 44 (1992).

${ }^{12}$ L. Shott, Phys. Fluids 30, 1795 (1987)

${ }^{13}$ K. Sato and F. Miyawaki, Phys. Fluids B 4, 1247 (1992).

${ }^{14}$ T. E. Sheridan, N. St. J. Braithwaite, and R. W. Boswell, Phys. Plasmas 6, 4375 (1999).

${ }^{15}$ T. E. Sheridan, Phys. Plasmas 8, 4240 (2001).

${ }^{16} \mathrm{~F}$. Valsaque, Ph.D. thesis, University of Nancy I, France (2002).

${ }^{17}$ K. U. Rieman, J. Phys. D 24, 493 (1991).

${ }^{18}$ G. Bachet, L. Cherigier, and F. Doveil, Phys. Plasmas 2, 1 (1995).

${ }^{19}$ L. Oksuz, M. Atta Kher, and N. Hershkowitz, Phys. Plasmas 8, 1729 (2001).

${ }^{20}$ G. D. Severn, Xu Wang, K. Eunsuk, and N. Hershkowitz, Phys. Rev. Lett. 90, 145001 (2003).

${ }^{21}$ X. Sun, S. A. Cohen, Earl E. Scime, and Mahmood Miah, Phys. Plasmas 12, 103509 (2005).

${ }^{22}$ R. J. Taylor, K. R. MacKenzie, and H. Ikezi, Rev. Sci. Instrum. 44, 726 (1978).

${ }^{23}$ R. L. Kurucz and B. Bell, Atomic Line Data, Kurucz CD-ROM No. 23, Smithsonian Astrophysical Observatory, Cambridge, MA (1995). 